\title{
State Estimation in Hybrid Systems With a Bounded Number of Mode Transitions
}

\author{
Daniel Sigalov \\ Technion - Israel Institute of Technology \\ Program for Applied Mathematics \\ Haifa 32000, Israel \\ dansigal@tx.technion.ac.il
}

\author{
Yaakov Oshman \\ Technion - Israel Institute of Technology \\ Department of Aerospace Engineering \\ Haifa 32000, Israel \\ yaakov.oshman@technion.ac.il
}

\begin{abstract}
We consider the problem of tracking the state of a hybrid system capable of performing a bounded number of mode switches. The system is assumed to follow either a nominal or an anomalous model, where the nominal model may stand for, e.g., the nonmaneuvering motion regime of a target or the fault-free operation mode of a sensor, and the anomalous model may stand for, e.g., the abrupt evasive maneuvers of a target or the faulty operation of a sensor. As is well known, the optimal algorithm requires implementation of an exponentially growing number of primitive Kalman filters. On the other hand, the system's switching dynamics is not Markov because of the a priori bounded number of model switches, thus ruling out the use of popular estimation schemes such as the interacting multiple model (IMM) and generalized pseudoBayesian (GPB) filters. We derive an efficient scheme that uses a number of primitive Kalman filters that is linear in the number of possible maneuvers. The scheme resembles the IMM algorithm in that it uses interaction between some of the primitive filters before every estimation cycle, thus reducing the number of such filters. The algorithm's performance is evaluated via a simulation study, and shown to outperform the state-of-the-art IMM filter in a typical example.
\end{abstract}

Keywords: Multiple model estimation, target tracking, hybrid systems, fault detection and identification.

\section{Introduction}

Hybrid systems are characterized by a continuously varying state vector and a discretely varying (switching) parameter vector, that takes values in some finite set [1]. The switching parameter vector is usually referred to as the system mode. The hybrid system framework is frequently used in multi-sensor fault-prone systems. Typical examples are navigation systems using the signals of global navigation satellite systems (that are prone to jamming and spoofing) and inertial sensors (accelerometers and rate gyros) that are, frequently, of low grade. Another typical application of the hybrid system framework is in maneuvering target tracking, where it is assumed that the target can maneuver, at any time, in one of a finite set of maneuvers (modes).

Since many hybrid systems are characterized by stochastic state and/or parameter vector, much work has been devoted to the simultaneous estimation of both state and parameters. As is well known, the mean squared optimal filtering algorithm for hybrid systems requires infinite computation resources [2]. Therefore, a variety of suboptimal estimation techniques was proposed [3-7]. In all of these cases, the number of mode switches permitted by the system's model was assumed to be unbounded, and the switching parameter vector was assumed to obey a Markovian transition law.

When the number of mode switches permitted by the system is bounded (assuming some knowledge about this bound), the Markovian assumption no longer holds. Typical problems featuring a bounded number of mode switches are encountered in maneuvering target tracking, particularly during the end-game phase of the interception scenario. Thus, [8] analyzes a stochastic ballistic missile interception scenario where the (incoming) blind theatre ballistic missile executes a random bang-bang maneuver consisting of a maximal maneuver in one direction, followed by a randomly-timed single switch to a maximal maneuver in the opposite direction. In [9], optimal differential games-based strategies are derived, showing that, for both adversaries, these are of the bang-bang type, with possibly a single direction change if the target has non-minimum phase dynamics. In [10], two possible target strategies are assumed: a constant maximum maneuver, and a square wave maneuver with a small number (one or two) of direction changes. Constant target maneuvers have also been assumed in [11].

This paper addresses the problem of tracking the state of a hybrid system capable of performing a bounded number of mode switches. It can be shown that the optimal tracking algorithm requires the imple- 
mentation of an exponentially growing number of primitive Kalman filters (KF), thus calling for suboptimal algorithms. On the other hand, the system's switching dynamics is not Markov because of the a priori bounded number of model switches, ruling out the use of popular estimation schemes such as the IMM [6] and GPB [2] algorithms. We derive an efficient estimation scheme that uses a number of primitive KFs that is linear in the number of possible maneuvers. The scheme resembles the IMM in that it uses interaction between the primitive filters before every estimation cycle, thus reducing the number of such filters. The algorithm's performance is evaluated via a simulation study, and shown to outperform the state-of-the-art IMM filter in a typical example. The study also demonstrates that the algorithm's performance degrades gracefully when the underlying assumptions do not hold.

The remainder of this paper is organized as follows. The problem is formulated in Section 2. Section 3 is devoted to the derivation of the new method. The algorithm steps are then summarized and the relation to IMM is discussed. In Section 4 we extend the filter to be capable of treating uncertainties in the initial model. The algorithm is tested and compared to IMM in Section 5. Concluding remarks are offered in Section 6.

\section{Problem Formulation}

Consider the standard state-space representation of a stochastic dynamical system

$$
\begin{aligned}
x_{k+1} & =A x_{k}+G w_{k} \\
z_{k} & =H x_{k}+v_{k} .
\end{aligned}
$$

Here $\left\{w_{k}\right\}$ and $\left\{v_{k}\right\}$ are mutually independent, zeromean white Gaussian noise sequences with covariances $Q$ and $R$, respectively. These processes are assumed to be independent of the initial state $x_{0}$, that is assumed to be Gaussian with mean $\bar{x}_{0}$ and covariance $P_{0}$.

The system (1)-(2) is specified by five matrices $A$, $H, G, Q$, and $R$, that may depend on the time index $k$. These matrices define the mode of the system, which we denote as $M_{k} \triangleq\left\{A_{k}, H_{k}, G_{k}, Q_{k}, R_{k}\right\}$. Different mode values may correspond to different flight regimes (e.g., maneuvering/non-maneuvering) of an aircraft, or different sensor conditions (e.g., nominal/faulty).

The state $x_{k}$ may be estimated optimally in the mean-square sense using a standard KF even if the mode sequence is time-dependent, as long as the exact value of $M_{k}$ is known for each $k$.

We consider the case where at time $k$ the mode $M_{k}$ may assume one of two possible values, $m_{1}$ or $m_{2}$. The model $m_{1}$ is the nominal one. In target tracking applications it represents the nominal, non-maneuvering motion regime of a target. In fault detection and identification (FDI) applications, it may represent the faultfree condition of the sensor. The second possible mode, $m_{2}$, represents the abnormal situation, e.g., a maneuvering target, or a faulty sensor. It is possible to describe various maneuver regimes using, e.g., Singer's exponentially correlated acceleration (ECA) model [12].

Consider the situation where the mode $M_{k}$ does not evolve in a deterministic manner. Namely, during the entire surveillance interval the target mode switches between the two models according to some stochastic law. We assume that the total number of mode switches is upper bounded by a known constant $r_{\max }$. Let $\left\{S_{k}\right\}$ be a sequence of random variables assuming the values $0, \ldots, r_{\max }$, such that $S_{k}=\ell$ if $\ell$ mode switches have occurred by time $k$. The switching mechanism considered here is described by the following transition law:

$$
\begin{aligned}
\operatorname{Pr} & \left\{M_{k+1}=m_{j} \mid M_{k}=m_{i}, S_{k}=\ell\right\} \\
& =\left\{\begin{array}{ll}
p_{i j} & \ell<r_{\max } \\
\delta_{i j} & \ell \geq r_{\max }
\end{array} \quad i, j \in\{1,2\}, k=0,1, \ldots\right.
\end{aligned}
$$

where $\left\{p_{i j}\right\}$ are known probabilities and $\delta_{i j}$ is Kronecker's delta. For clarity of exposition, we assume that $\operatorname{Pr}\left\{M_{0}=m_{1}\right\}=1$. This assumption will be relaxed in section 4. Equations (1), (2) and (3) define a hybrid stochastic system as the continuous uncertainty associated with $x_{k}$ is accompanied by a discrete uncertainty associated with the (discrete) random mode transition law.

It is well known that the optimal minimum mean squared error (MMSE) estimate of the state $x_{k}$ using the measurement sequence $Z_{k} \triangleq\left\{z_{0}, \ldots, z_{k}\right\}$ is given by the conditional expectation $\mathbb{E}\left[x_{k} \mid Z_{k}\right]$. It can be shown that the optimal filter for computing $\hat{x}_{k}=\mathbb{E}\left[x_{k} \mid Z_{k}\right]$ requires the use of (primitive) KFs, in a number that grows polynomially in time and exponentially in $r_{\max }$. Thus, applying the optimal scheme for such systems cannot be practical even in short-duration scenarios.

Although the number of mode switches constitutes a Markov chain with state space $\left\{0,1, \ldots, r_{\max }\right\}$, the above system is not a standard Markov-jump linear system (MJLS), because the mode transition law is Markov only given the number of mode switches that have occurred so far. Thus, the IMM and related methods cannot be applied directly to the problem at hand. The IMM assumes that modes switch according to a Markov law, such that transition probabilities are captured by a known transition probability matrix (TPM). Because the process is not Markov, such a matrix does not exist, and, therefore, applying the IMM and related algorithms can only be done in an approximate manner by defining a pseudo-transition matrix. Note that for the case $r_{\max }=1$ the mode transition law is Markov, since the number of occurred switches (0 or 1$)$ uniquely determines the current mode (assuming $M_{0}=m_{1}$ ).

Our goal is to obtain an efficient suboptimal algorithm capable of tracking the state $x_{k}$ of a system that 
may perform a bounded number of mode switches, using the measurements $Z_{k} \triangleq\left\{z_{0}, \ldots, z_{k}\right\}$, without assuming Markovian mode transitions.

\section{Algorithm Derivation}

We assume, for simplicity, $p_{12}=p_{21}=p$. Generalization to arbitrary values of the transition probabilities is straightforward and the derivation is omitted.

The optimal estimate of the state $\hat{x}_{k}=\mathbb{E}\left[x_{k} \mid Z_{k}\right]$ is the mean of the posterior density $\mathrm{p}\left(x_{k} \mid Z_{k}\right)$. We have

$$
\begin{aligned}
\mathrm{p}\left(x_{k} \mid Z_{k}\right) & =\sum_{\ell=0}^{r_{\max }} \mathrm{p}\left(x_{k} \mid Z_{k}, S_{k}=\ell\right) \operatorname{Pr}\left\{S_{k}=\ell \mid Z_{k}\right\} \\
& \triangleq \sum_{\ell=0}^{r_{\max }} \mathrm{p}\left(x_{k} \mid z_{k}, Z_{k-1}, S_{k}=\ell\right) \mu_{\ell}(k) .
\end{aligned}
$$

Equation (4) can be viewed as an implied definition of the switching sequence probabilities $\left\{\mu_{\ell}(k)\right\}_{\ell=0}^{r_{\max }}$, which serve here as weighting factors. In the sequel we separately compute these weighting factors along with the conditional posterior pdf's, $\mathrm{p}\left(x_{k} \mid z_{k}, Z_{k-1}, S_{k}=i\right)$.

\subsection{Weighting Factors}

At each time instance $k$ the number of mode switches is upper bounded both by $r_{\max }$, by the problem's definition, and, naturally, by $k$. Using Bayes' rule and the law of total probability we have, for $1 \leq \ell \leq \min \left(r_{\max }, k\right)$ and some normalization constant $c$,

$$
\begin{aligned}
\mu_{\ell}(k) & =\operatorname{Pr}\left\{S_{k}=\ell \mid Z_{k}\right\} \\
= & \frac{1}{c} \mathrm{p}\left(z_{k} \mid Z_{k-1}, S_{k}=\ell\right) \operatorname{Pr}\left\{S_{k}=\ell \mid Z_{k-1}\right\} \\
= & \frac{1}{c} \mathrm{p}\left(z_{k} \mid Z_{k-1}, S_{k}=\ell\right) \\
& \times \sum_{j=\ell-1}^{\ell} \operatorname{Pr}\left\{S_{k}=\ell \mid S_{k-1}=j, Z_{k-1}\right\} \mu_{j}(k-1) \\
= & \frac{1}{c} \mathrm{p}\left(z_{k} \mid Z_{k-1}, S_{k}=\ell\right) \\
& \times\left[p \mu_{\ell-1}(k-1)+(1-p) \mu_{\ell}(k-1)\right] .
\end{aligned}
$$

For $\ell=0$ the equation for $\mu_{\ell}(k)$ is modified accordingly, yielding, along with (5),

$$
\begin{aligned}
\mu_{\ell}(k)= & \frac{1}{c} \mathrm{p}\left(z_{k} \mid Z_{k-1}, S_{k}=\ell\right) \\
& \times\left[p \mu_{\ell-1}(k-1) \delta_{\ell 0}+(1-p) \mu_{\ell}(k-1)\right] .
\end{aligned}
$$

The likelihood $\mathrm{p}\left(z_{k} \mid Z_{k-1}, S_{k}=\ell\right)$ may be approximated to be Gaussian, as follows

$$
\mathrm{p}\left(z_{k} \mid Z_{k-1}, S_{k}=\ell\right) \approx \mathcal{N}\left(z_{k} ; \hat{z}_{k}^{\ell}, S_{k}^{\ell}\right)
$$

where $\hat{z}_{k}^{\ell}$ and $S_{k}^{\ell}$ are the predicted measurement and innovation covariance, respectively, that are computed by a $\mathrm{KF}$ matched to the event that $\ell$ switches have occurred by the time $k$. The Gaussian approximation (7) is commonly used in the target tracking literature, and especially in suboptimal filters for hybrid systems, such as the GPB [2] and IMM [6,13] filters.

\subsection{Conditional Posterior Densities}

Consider the posterior densities in (4), conditioned on the number of mode switches. Using Bayes' rule

$$
\begin{aligned}
& \mathrm{p}\left(x_{k} \mid z_{k}, Z_{k-1}, S_{k}=\ell\right) \\
& \quad=\frac{\mathrm{p}\left(z_{k} \mid x_{k}, S_{k}=\ell\right)}{\mathrm{p}\left(z_{k} \mid Z_{k-1}, S_{k}=\ell\right)} \mathrm{p}\left(x_{k} \mid Z_{k-1}, S_{k}=\ell\right),
\end{aligned}
$$

where we have used the fact that, conditioned on the event $\left\{S_{k}=\ell\right\}$, the probabilistic law of $z_{k}$ is completely determined by $x_{k}$ and is, thus, independent of $Z_{k-1}$.

Eq. (8) is the measurement update step of a filter conditioned on $\ell$ switches. Starting with the prior, $\mathrm{p}\left(x_{k} \mid Z_{k-1}, S_{k}=\ell\right)$, it represents the evolution of the density after processing the new measurement, $z_{k}$.

To perform the time update step we expand the prior density using the law of total probability:

$$
\begin{aligned}
& \mathrm{p}\left(x_{k} \mid S_{k}=\ell, Z_{k-1}\right) \\
&=\sum_{j=\ell-1}^{\ell} \mathrm{p}\left(x_{k} \mid S_{k}=\ell, S_{k-1}=j, Z_{k-1}\right) \\
& \quad \times \operatorname{Pr}\left\{S_{k-1}=j \mid S_{k}=\ell, Z_{k-1}\right\} .
\end{aligned}
$$

Clearly, one cannot compute the density $\mathrm{p}\left(x_{k} \mid S_{k}=\right.$ $\left.\ell, S_{k-1}=j, Z_{k-1}\right)$ precisely without resorting to exhaustive enumeration. Thus, it is approximated by merging the previous histories into a single estimate:

$$
\begin{gathered}
\mathrm{p}\left(x_{k} \mid S_{k}=\ell, Z_{k-1}\right) \\
\approx \sum_{j=\ell-1}^{\ell} \mathrm{p}\left(x_{k} \mid S_{k}=\ell, S_{k-1}=j,\left\{\hat{x}_{k-1}^{i}, P_{k-1}^{i}\right\}_{i=0}^{r_{\max }}\right) \\
\quad \times \operatorname{Pr}\left\{S_{k-1}=j \mid S_{k}=\ell, Z_{k-1}\right\} .
\end{gathered}
$$

In other words, the measurement history $Z_{k-1}$ is replaced by a function of it, represented here by the set of conditioned estimates and their corresponding covariances. Defining $\mu_{j \mid \ell}(k-1 \mid k) \triangleq$ $\operatorname{Pr}\left\{S_{k-1}=j \mid S_{k}=\ell, Z_{k-1}\right\},(10)$ is rewritten as

$$
\begin{aligned}
& \mathrm{p}\left(x_{k} \mid S_{k}=\ell, Z_{k-1}\right) \\
& \quad \sum_{j=\ell-1}^{\ell} \mathrm{p}\left(x_{k} \mid S_{k}=\ell, S_{k-1}=j,\left\{\hat{x}_{k-1}^{i}, P_{k-1}^{i}\right\}_{i=0}^{r_{\max }}\right) \\
& \quad \times \mu_{j \mid \ell}(k-1 \mid k) .
\end{aligned}
$$

Each of the (two) densities in (11) is approximated as Gaussian with parameters determined by the latest active model and, therefore, each term is completely specified by the event $\left\{S_{k-1}=j\right\}$, in the following manner:

$$
\begin{aligned}
\mathrm{p}\left(x_{k} \mid\right. & \left.S_{k}=\ell, S_{k-1}=j,\left\{\hat{x}_{k-1}^{i}, P_{k-1}^{i}\right\}_{i=0}^{r_{\max }}\right) \\
& \approx \mathcal{N}\left(x_{k} ; \mathbb{E}\left[x_{k} \mid S_{k}=\ell, \hat{x}_{k-1}^{j}\right], P_{k-1}^{j}\right) .
\end{aligned}
$$


Summarizing, the prior density is approximated as

$$
\begin{aligned}
& \mathrm{p}\left(x_{k} \mid S_{k}=\ell, Z_{k-1}\right) \\
& \approx \sum_{j=\ell-1}^{\ell} \mathcal{N}\left(x_{k} ; \mathbb{E}\left[x_{k} \mid S_{k}=\ell, \hat{x}_{k-1}^{j}\right], P_{k-1}^{j}\right) \\
& \quad \times \mu_{j \mid \ell}(k-1 \mid k),
\end{aligned}
$$

which is a Gaussian mixture that can be further approximated by a single Gaussian with the same first and second moments. Namely,

$$
\mathrm{p}\left(x_{k} \mid S_{k}=\ell, Z_{k-1}\right) \approx \mathcal{N}\left(x_{k} ; m_{\ell}, \Sigma_{\ell}\right)
$$

with

$$
\begin{aligned}
m_{\ell}= & \mathbb{E}\left[x_{k} \mid S_{k}=\ell, \hat{x}_{k-1}^{\ell-1}\right] \mu_{\ell-1 \mid \ell}(k-1 \mid k) \\
& +\mathbb{E}\left[x_{k} \mid S_{k}=\ell, \hat{x}_{k-1}^{\ell}\right] \mu_{\ell \mid \ell}(k-1 \mid k)
\end{aligned}
$$

and

$$
\begin{aligned}
& \Sigma_{\ell} \\
& =\left(P_{k-1}^{\ell-1}+\left(\hat{x}_{k-1}^{\ell-1}-m_{\ell}\right)\left(\hat{x}_{k-1}^{\ell-1}-m_{\ell}\right)^{T}\right) \mu_{\ell-1 \mid \ell}(k-1 \mid k) \\
& +\left(P_{k-1}^{\ell}+\left(\hat{x}_{k-1}^{\ell}-m_{\ell}\right)\left(\hat{x}_{k-1}^{\ell}-m_{\ell}\right)^{T}\right) \mu_{\ell \mid \ell}(k-1 \mid k) .
\end{aligned}
$$

Eq. (15a) has the following interpretation. Starting with the previously obtained conditioned estimates, $\hat{x}_{k-1}^{\ell-1}$ and $\hat{x}_{k-1}^{\ell}$, they are propagated through the $\ell-\mathrm{KF}$ to obtain the time updated estimates, $\mathbb{E}\left[x_{k} \mid S_{k}=\ell, \hat{x}_{k-1}^{\ell-1}\right]$ and $\mathbb{E}\left[x_{k} \mid S_{k}=\ell, \hat{x}_{k-1}^{\ell}\right]$, respectively. These are then fused into a single estimate using the mixing probabilities $\mu_{\ell-1 \mid \ell}(k-1 \mid k)$ and $\mu_{\ell \mid \ell}(k-1 \mid k)$. Since the KF is a linear operator, the fusion of the estimates can be performed before the conditional time update step, and the mean of the Gaussian density in (15a) may be rewritten as

$m_{\ell}=\mathbb{E}\left[x_{k} \mid S_{k}=\ell, \hat{x}_{k-1}^{\ell-1} \mu_{\ell-1 \mid \ell}(k-1 \mid k)+\hat{x}_{k-1}^{\ell} \mu_{\ell \mid \ell}(k-1 \mid k)\right]$

Using (16) in (14) we obtain the following property of the proposed algorithm. The input (i.e., the measurement updated estimate from the previous cycle) to the filter matched to $\ell$ mode switches is obtained from an interaction of the outputs of itself and of the filter matched to $\ell-1$ mode switches. The interaction is performed by combining these outputs using the mixing probabilities $\mu_{\ell-1 \mid \ell}(k-1 \mid k)$ and $\mu_{\ell \mid \ell}(k-1 \mid k)$. Clearly, the filter matched to $\ell=0$ switches does not interact with any other filter, and, in fact, operates as a usual KF matched to the model $m_{1}$.

It remains to describe the computation of the mixing probabilities, $\mu_{j \mid \ell}(k-1 \mid k)$. This is done next.

\subsection{Mixing Probabilities}

Using Bayes' rule yields, for some constant $c$,

$$
\begin{aligned}
\operatorname{Pr} & \left\{S_{k-1}=j \mid S_{k}=\ell, Z_{k-1}\right\} \\
& =\frac{1}{c} \operatorname{Pr}\left\{S_{k}=\ell \mid S_{k-1}=j, Z_{k-1}\right\} \mu_{j}(k-1) .
\end{aligned}
$$

Note that $\operatorname{Pr}\left\{S_{k}=\ell \mid S_{k-1}=j, Z_{k-1}\right\}$ does not vanish only for $j=\ell$ and $j=\ell-1$. It is equal to $1-p$ in the former case, as no mode switch occurred at time $k-1$, and to $p$ in the latter case. Thus, the mixing probability recursions are summarized as follows:

$$
\mu_{j \mid \ell}(k-1 \mid k)=\frac{1}{c}\left\{\begin{array}{ll}
p \mu_{\ell-1}(k-1) & j=\ell-1 \\
(1-p) \mu_{\ell}(k-1) & j=\ell
\end{array} .\right.
$$

It is worth mentioning that this recursive computation does not entail any approximation.

\subsection{Algorithm Summary and Filter Structure}

For convenience, the algorithm is summarized in $\mathrm{Al}-$ gorithm 1.

Algorithm 1

Input: $Z_{k}=\left\{z_{1}, \ldots, z_{k}\right\}, m_{1}, m_{2}, \hat{x}_{0}, P_{0}$

1: Mixing Probabilities: Compute $\mu_{j \mid \ell}(k-1 \mid k), \ell=$ $0,1, \ldots, \min \left(k, r_{\max }\right)$ using Eq. (18).

2: Mixing: Given the outputs of the filters $\ell$ and $\ell-1$ compute

$$
\hat{x}_{0}^{\ell}=\hat{x}_{k-1}^{\ell-1} \mu_{\ell-1 \mid \ell}(k-1 \mid k)+\hat{x}_{k-1}^{\ell} \mu_{\ell \mid \ell}(k-1 \mid k)
$$

for $\ell=0, \ldots, \min \left(k, r_{\max }\right)$ and the associated covariances using (15b).

3: Conditional Filtering: Using $\hat{x}_{0}^{\ell}$ and $(15 \mathrm{~b})$ compute the posterior estimates $\hat{x}_{k}^{\ell}$ and $P_{k}^{\ell}$ as well as the conditional likelihoods given in Eq. (7).

4: Probabilities Update: Compute the weighting factors of (4) using (6).

5: Output: Compute $\hat{x}_{k}$ (the mean of (4)) with the corresponding covariance.

Output: $\hat{x}_{k}, P_{k}$.

The schematic structure of the proposed filter is presented in Fig. 1. Notice that each filter interacts with at most one filter to produce the initial condition for the next iteration. Similarly to other multiple model estimation methods, the final estimates are used for output purposes only, and are not part of the estimation cycle.

\subsection{Relation to IMM}

Despite the conceptual similarity between the proposed algorithm and the IMM, which is exhibited by the cooperation of the primitive filters to obtain an approximation of the first two moments of the desired posterior density $\mathrm{p}\left(x_{k} \mid Z_{k}\right)$, there are several important differences that we outline below.

First, each primitive KF in the IMM algorithm is matched to one of the specific models the system is assumed to obey. In the new algorithm, on the other hand, each KF is matched to a different event describing the number of mode switches that have occurred. Since 

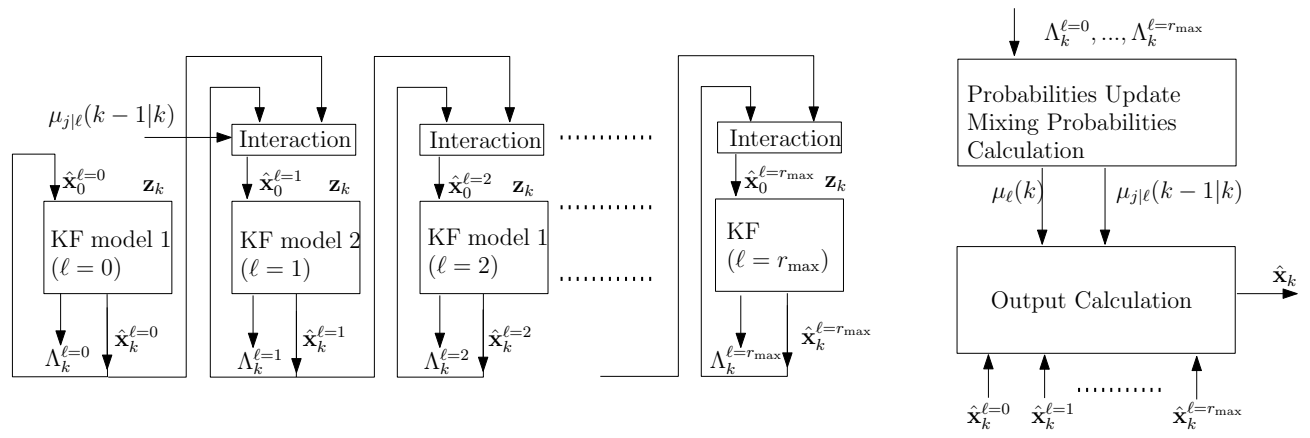

Figure 1. Schematic implementation of the proposed filter (the covariances accompanying the estimates are not shown, for clarity).

an even number of mode switches does not affect the actual mode of the system, half of the primitive filters are matched to $m_{1}$ and the other half to $m_{2}$. It is worth noting that, although there is some redundancy in that the same filter is duplicated several times, direct savings cannot be obtained, as each of the (same) filters is fed with different inputs at the same estimation cycle.

The second difference is related to the filter cooperation mechanism. Whereas in the IMM algorithm all outputs are used in the mixing stage to produce the initial conditions of each of the filter, in the new algorithm, the input of filter $\ell$ is produced by mixing the outputs of only two filters, $\ell$ and $\ell-1$. The initial conditions are unaffected by other KFs. This difference stems from the mode transition mechanism - at each time the number of mode switches may either stay unchanged, or increase by one.

We note, in passing, that the problem may be reformulated using a Markovian framework by introducing a super mode $\left\{S_{k}, M_{k}\right\}$ that consists of the actual mode and the number of performed transitions. This would allow running the standard IMM, however, at the expense of a significantly increased number of primitive KFs running in parallel.

\section{Unknown Initial Model}

The filter derived in Section 3 assumes that the initial model at time $k=0$ is $m_{1}$. In some applications this might be an unrealistic stipulation. We now extend the basic algorithm to handle the case of an unknown initial model.

Consider, again, the optimal estimate of the state $x_{k}$ given by the conditional expectation $\mathbb{E}\left[x_{k} \mid Z_{k}\right]$. Conditioning on the initial model yields

$$
\begin{gathered}
\mathbb{E}\left[x_{k} \mid Z_{k}\right]=\mathbb{E}\left[x_{k} \mid Z_{k}, M_{0}=m_{1}\right] \operatorname{Pr}\left\{M_{0}=m_{1} \mid Z_{k}\right\} \\
+\mathbb{E}\left[x_{k} \mid Z_{k}, M_{0}=m_{2}\right] \operatorname{Pr}\left\{M_{0}=m_{2} \mid Z_{k}\right\} .
\end{gathered}
$$

In principle, both conditional expectations in Eq. (19) assume known initial models, and, therefore, can be computed using the basic algorithm of Section 3 (with a straightforward modification). It remains to compute the conditional probability $\operatorname{Pr}\left\{M_{0}=m_{1} \mid Z_{k}\right\}$, which amounts to identification of $M_{0}$. This can be done sequentially as $\alpha_{k} \triangleq \operatorname{Pr}\left\{M_{0}=m_{1} \mid Z_{k}\right\}=$ $\frac{1}{c} \mathrm{p}\left(z_{k} \mid Z_{k-1}, M_{0}=m_{1}\right) \alpha_{k-1}$, meaning that the solution of the identification problem boils down to computing the model-matched innovation density $\mathrm{p}\left(z_{k} \mid Z_{k-1}, M_{0}=m_{1}\right)$. This density may be computed either by conditioning on $\left\{S_{k}=\ell\right\}$, or by performing a likelihood ratio-based test using the ratio $\mathrm{p}\left(z_{k} \mid Z_{k-1}, M_{0}=m_{1}\right) / \mathrm{p}\left(z_{k} \mid Z_{k-1}, M_{0}=m_{2}\right)$.

\section{Simulation Study}

In this section we test the performance of the proposed algorithm. For better visualization, and without loss of generality, even numbers of mode switches are taken in the examples, such that the final model is $m_{1}$.

In all figures in the sequel, horizontal axes represent time in seconds. Position, velocity and acceleration errors are in meters, meters per second, and meters per second squared, respectively.

\subsection{General Simulation Setup}

We consider the following dynamical system

$$
x_{k+1}=A_{k}^{i} x_{k}+G_{k}^{i} w_{k}^{i}, i=1,2,
$$

where the state vector $x_{k}=\left[\begin{array}{lll}p_{k} & v_{k} & a_{k}\end{array}\right]^{T}$ comprises the target's position, velocity, and acceleration.

The nominal model $m_{1}$ is the discrete white noise acceleration model [13] with the associated process noise variance $\sigma_{w_{1}}^{2}$. The abnormal mode, $m_{2}$, is chosen to be the discrete Wiener process acceleration model [13] accompanied with the covariance $\sigma_{w_{2}}^{2}$.

The measurements are generated according to (2) where $H=\left[\begin{array}{lll}1 & 0 & 0\end{array}\right]$, and $v_{k} \sim \mathcal{N}\left(0, \sigma_{v}^{2}\right)$. In all examples below we have used $\sigma_{w_{1}}^{2}=0.3, \sigma_{w_{2}}^{2}=6, \sigma_{v}^{2}=140$.

\subsection{Example: $r_{\max }=2$}

The following scenario, lasting 400 seconds is considered. Starting at $x_{0}=\left[\begin{array}{lll}0 & 0 & 0\end{array}\right]^{T}$ the target performs two 
mode switches according to the following pattern. 1) For the first 100 seconds the target moves according to $\left.m_{1}, 2\right)$ at $k=100$ the target switches to $m_{2}$ and complies with this model for additional 100 seconds, 3) at $k=200$, the target switches back to $m_{1}$. Thus, in this (deterministic) scenario, mode switches occur after 100 and 200 seconds. To comply with the assumed model we set $p_{12}=p_{21}=p=0.01$.

The new algorithm is compared with the performance of the IMM filter. Since with $r_{\max }>1$ the mode transition dynamics is not Markovian, the transition probability matrix of the IMM needs to be adjusted in some manner. According to our modeling, a reasonable choice would be TPM $=\left(\begin{array}{lll}p_{11} & p_{12} \\ p_{21} & p_{22}\end{array}\right)$, where we set $p_{12}=p_{21}=p=0.01$, to comply with a mode switch once in every 100 seconds, on average.

As an overall reference we also generate the optimal estimate using a "genie-based" KF that knows the exact times of the mode switches. Knowing the model at each time degenerates the problem to the standard setting such that a standard KF yields optimal results.

Fig. 2 presents the squared errors of the position and velocity of the new filter, the IMM filter and the geniebased KF, averaged over 200 Monte Carlo runs. It is readily seen that before the second (and last) switch both the new filter and the IMM yield similar errors. However, after the second switch the performance of the new filter is superior to that of IMM. In fact, the errors associated with the new filter closely approximate those of the genie-based KF, thus achieving the best possible performance.

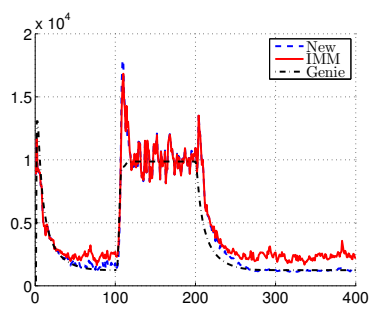

(a) Position

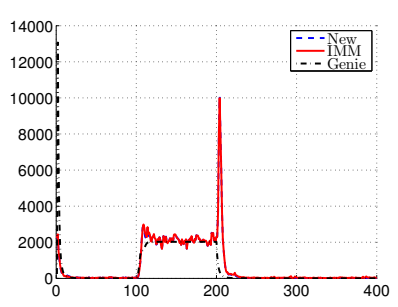

(c) Velocity (b) Position (Zoom)

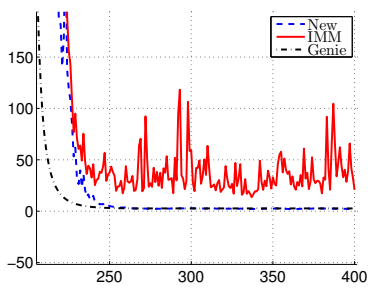

(d) Velocity (Zoom)

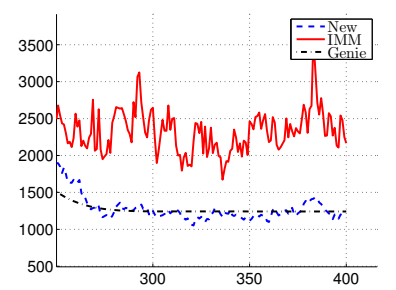

Figure 2. Position and velocity errors of the new filter, IMM, and genie-based KF, $r_{\max }=2$.

The superiority of the proposed method after the maximum number of model switches has been exhausted, is best understood via observing Fig. 3, showing the probabilities of the number of switches as computed by the proposed algorithm and the mode probabilities computed by the IMM. Notice that after

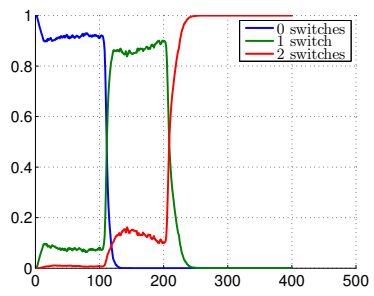

(a) Proposed filter

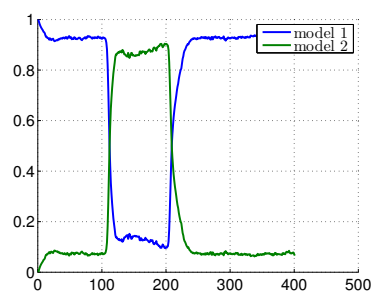

(b) IMM
Figure 3. Probabilities of number of switches and mode probabilities of IMM, $r_{\max }=2$.

the maximum number of switches, $r_{\max }$, has been exhausted, the new algorithm knows precisely the model being currently in effect. Therefore, it nullifies all probabilities $\mu_{\ell}$ for $\ell<r_{\max }$ and assigns $\mu_{r_{\max }}=1$. This means that the scheme's state estimate is based on the true model, which yields optimal performance. The IMM filter, on the other hand, assumes that the mode transitions obey a Markovian structure, which is not true in our case. Therefore it cannot nullify any of the modal probabilities in order to keep both models 'alive' (so as to be prepared for a possible future switch). This introduces an additional mismatched term to the output estimate, thus increasing the estimation error.

\subsection{Monte Carlo Evaluation}

In this experiment we test the algorithms in a completely random scenario. The simulation setup is similar to that of the previous examples, except that now the switching times are generated in a random manner. Therefore, the MSEs are the true squared errors averaged over all the randomness in the system (and not only over the measurement and process noises as done in the previous example). The duration of the scenario is increased to 800 seconds, the maximum number of mode switches is taken to be 2 , and the switching probability is chosen to be $p=0.004$. Thus, with a high probability, both switches occur at the beginning of the scenario (within the first 500 seconds, on average) and in the final part of $\approx 300$ seconds the target continues its motion according to $m_{1}$. For easier visualization, we present the mean squared errors of the position and velocity normalized by those obtained by the genie-based KF. Fig. 4 shows the position and velocity estimation normalized MSEs for both the new filter and the IMM filter. The results are averaged over 500 independent runs. The new algorithm's errors are 


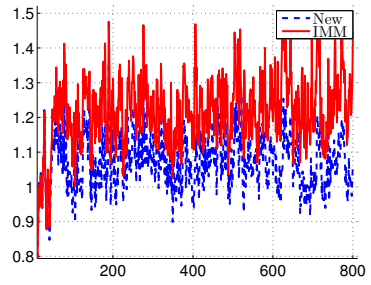

(a) Position

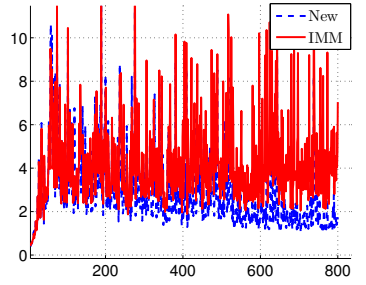

(b) Velocity
Figure 4. Normalized estimation errors of the new filter and IMM, Monte Carlo evaluation.

only slightly higher than those of the genie-based KF, meaning that it achieves nearly optimal performance. In addition, it is clear the new filter outperforms the IMM filter. The explanation for these results is similar to the one given in the previous example. In the second half of the time interval both mode switches have already occurred, and the target is driven according to the $m_{1}$ dynamics. Knowing the maximum number of switches, the new filter generates estimates obtained by $m_{1}$, the correct model. In contradistinction, the IMM filter is forced to maintain the second model as well, thus increasing the overall errors.

\subsection{Robustness}

We now test the performance when the assumption on the total number of switches is incorrect by assuming $r_{\max }$ that is higher or lower than the actual one.

The same dynamic models are used as in the previous examples, with a scenario duration of 500 seconds. The target performs 4 model switches with the exact pattern being as follows. 1) During the subintervals [0 100], [200 300], [400 500] the target maneuvers according to $\left.m_{1}, 2\right)$ during the subintervals [100 200], [300 400] the target follows the model $m_{2}$.

\subsubsection{Overestimated $r_{\max }$}

We set $r_{\max }=6$, whereas the true value is 4 . The position and velocity errors, normalized by those of the genie-based KF, are presented in Fig. 5. The corresponding probabilities are presented in Fig. 6. It is readily seen that, although the desired effect of degenerating the probabilities to zero and one does not happen anymore (since the predefined number of switches has not occurred), the performance of the new algorithm is comparable to that of the IMM with some superiority at the end of nonmaneuvering sections. Namely, even for the case of overestimated number of maneuvers, the performance gracefully degrades and remains tolerable and even superior to that of the IMM (at the expense, however, of additional computation burden in terms of the extra filters running simultaneously). Notice also

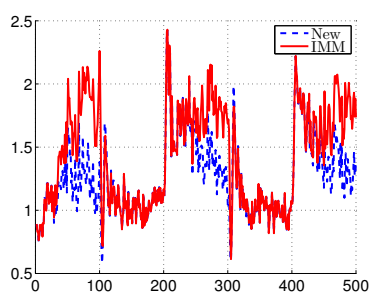

(a) Position

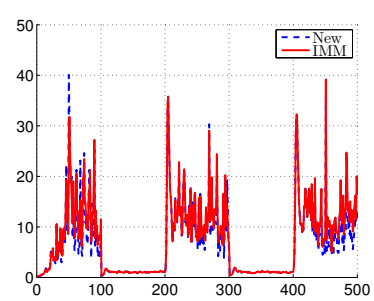

(b) Velocity
Figure 5. Normalized estimation errors of the new filter and IMM, overestimated $r_{\max }$.

that the probabilities of 5 and 6 switches remain low throughout the entire scenario.

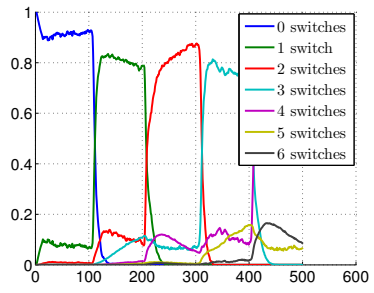

(a) Proposed filter

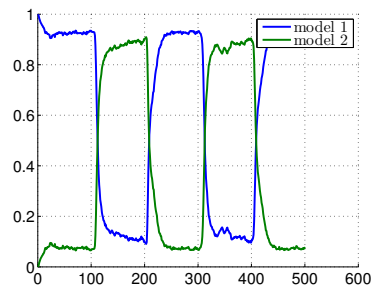

(b) IMM
Figure 6. Probabilities of number of switches and IMM mode probabilities, overestimated $r_{\max } \cdot$

\subsubsection{Underestimated $r_{\max }$}

In this experiment we set $r_{\max }=2$, whereas the true value remains 4 . The position and velocity errors for this case, normalized by those of the genie-based KF, are presented in Fig. 7. The corresponding probabilities are presented in Fig. 8. A priori one could expect a complete crash of the algorithm. However, this is not the case. Until the second mode switch (inclusive), the algorithm behaves as previously, and after that the corresponding probability degenerates to 1 as no additional switches are expected. The corresponding errors are lower than those of the IMM due to the reasons outline in the previous examples. The nonconservativeness of the algorithm causes a significant position error at $k \approx 300$, when the third mode switch occurs (as opposed to the assumption). However, this singular error is reduced quickly and the algorithm coincides again with the IMM. During this interval, the dominating filter is the one corresponding to $m_{1}$ and, therefore, the probability of 1 switch rises again. It should, however, be interpreted as the probability of 3 occurred switches. After the fourth switch the situation is identical to that after the second one - the algorithm 


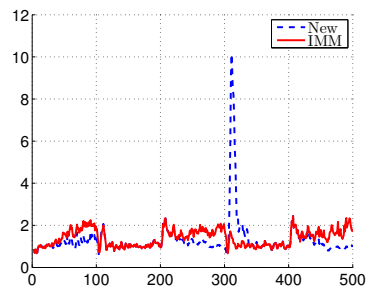

(a) Position

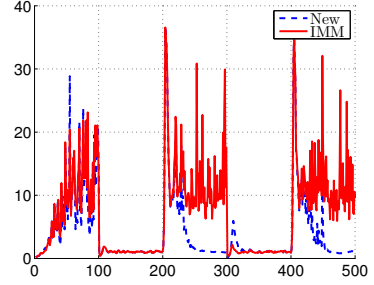

(b) Velocity
Figure 7. Normalized estimation errors of the new filter and IMM, underestimated $r_{\max }$.

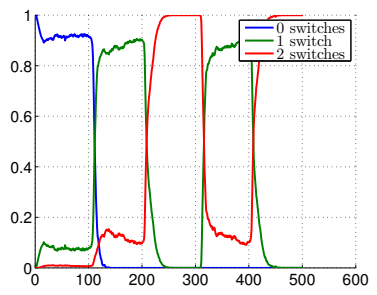

(a) Proposed filter

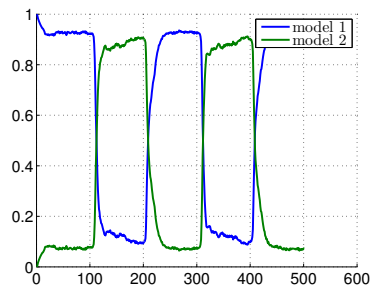

(b) IMM
Figure 8. Probabilities of number of switches and IMM mode probabilities, underestimated $r_{\max }$.

'believes' that all switches are exhausted, thus raising the corresponding probability to almost 1 , and outperforming the IMM filter in terms of estimation errors.

\section{Conclusions}

We have proposed a novel efficient algorithm for tracking the state of a hybrid system characterized by a bounded number of mode switches. Unlike in classical multiple model estimation problems, model transitions do not possess Markovian dynamics, and direct utilization of popular methods such as the IMM and GPBr filters is inadmissible. The new filter inherits several properties of the state-of-the-art IMM filter, such as interaction of primitive KFs, constituting the entire estimation scheme at each cycle. Except for the trivial case where the target is limited to performing just one maneuver, the proposed filter is superior to ad-hoc utilization of the IMM filter, as demonstrated via several numerical examples. A simulation study shows that the performance of the new filter gracefully degrades when the number of switches is either over- or underestimated relative to the true number of switches.

\section{Acknowledgement}

The authors wish to thank an anonymous reviewer for pointing out an additional relation to IMM.

\section{References}

[1] E.-K. Boukas and Z.-K. Liu, Deterministic and Stochastic Time-Delay Systems. Boston: Birkhäuser, 2002.

[2] G. Ackerson and K. Fu, "On state estimation in switching environments," IEEE Trans. on Automatic Control, vol. 15, no. 1, pp. 10-17, 1970.

[3] N. Nahi, "Optimal recursive estimation with uncertain observation," IEEE Trans. on Information Theory, vol. IT-15, no. 4, pp. 457-462, July 1969.

[4] A. Jaffer and S. Gupta, "Optimal sequential estimators of discrete processes with markov interrupted observations," IEEE Trans. on Information Theory, vol. IT-17, no. 6, pp. 614-616, September 1971.

[5] J. K. Tugnait, "Detection and Estimation for Abruptly Changing Systems," Automatica, vol. 18, no. 5, pp. 607-615, 1982.

[6] H. Blom and Y. Bar-Shalom, "The interacting multiple model algorithm for systems with Markovian switching coefficients," IEEE Trans. on Automatic Control, vol. 33, no. 8, pp. 780-783, 1988.

[7] N. de Freitas, "Rao-Blackwellised Particle Filtering for Fault Diagnosis," in Proc. of 2002 IEEE Aerospace Conference, Big Sky, MT, Mar. 2002, pp. $4-1767-4-1772$.

[8] T. Shima, Y. Oshman, and J. Shinar, "Efficient multiple model adaptive estimation in ballistic missile interception scenarios," Journal of Guidance, Control, and Dynamics, vol. 25, no. 4, pp. 667-675, 2002.

[9] T. Shima, "Capture conditions in a pursuit-evasion game between players with biproper dynamics," Journal of Optimization: Theory and Applications, vol. 126, no. 3, pp. 503-528, September 2005.

[10] V. Shaferman and T. Shima, "Linear quadratic guidance laws for imposing a terminal intercept angle," Journal of Guidance, Control, and Dynamics, vol. 31, no. 5, pp. 1400-1412, 2008.

[11] T. Shima, "Deviated velocity pursuit," in Proceedings of the AIAA Guidance, Navigation, and Control Conference, Hilton Head, SC, AIAA. AIAA, August 2007.

[12] X. Li and V. Jilkov, "Survey of maneuvering target tracking. Part I: Dynamic models," IEEE Trans. on Aerospace and Electronic Systems, vol. 39, no. 4, p. 1333, 2003.

[13] Y. Bar-Shalom, X. Li, and T. Kirubarajan, Estimation with Applications to Tracking and Navigation. New York: Wiley, 2001. 